\title{
Comments on the Numerical Simulation of Homogeneous Stably-Stratified Turbulence
}

\author{
Sergej S. Zilitinkevich
}

Received: 22 November 2009 / Accepted: 25 February 2010 / Published online: 20 March 2010

(C) The Author(s) 2010. This article is published with open access at Springerlink.com

\begin{abstract}
The standard design for the direct numerical simulation of homogeneous stably-stratified turbulence assumes that the simulated turbulence is fully characterised by the gradient Richardson number. This assumption is justified only in sufficiently strong stratification when the Obukhov turbulent length scale, $L$, is essentially smaller than the depth of the computational domain, $H$. Otherwise simulations are not quite realistic because they cut off the large-scale part of the turbulence spectrum, namely, the scales comparable with or larger than $H$ but smaller than $L$, that is just the eddies most sensitive to the stratification.
\end{abstract}

Keywords Applicability limit · Depth of computational domain ·

Direct numerical simulation · Stably stratified turbulence $\cdot$ Turbulent length scale

\section{Introduction}

Since Gerz et al. (1989) and Schumann and Gerz (1995), the standard design of the eddy-resolving numerical simulation of stationary homogeneous stably-stratified turbulence prescribes height-constant values of the mean shear, $S=d \bar{u} / d z$, and mean density gradient, $d \bar{\rho} / d z$, in a domain of height $H$, and it is assumed that the simulated turbulence is fully characterised by the gradient Richardson number:

$$
R i \equiv(N / S)^{2},
$$

\section{S. S. Zilitinkevich $(\varangle)$}

Meteorological Research, Finnish Meteorological Institute, P.O. Box 503, 00101 Helsinki, Finland e-mail: sergej.zilitinkevich@fmi.fi

\section{S. S. Zilitinkevich}

Nansen Environmental and Remote Sensing Centre/Bjerknes Centre for Climate Research, Bergen, Norway

\section{S. S. Zilitinkevich}

Department of Physics, Division of Atmospheric Sciences and Geophysics, University of Helsinki, Helsinki, Finland 
where $z$ is the height, $\bar{u}$ is the mean velocity, $N^{2}=\beta d \bar{\rho} / d z$ is the squared Brunt-Väisälä frequency, $\beta=g / \bar{\rho}_{0}$ is the buoyancy parameter, $g$ is the acceleration of gravity, and $\bar{\rho}_{0}$ is a reference value of density.

Generally, the simulated turbulence is controlled also by $H$ but the stable stratification imposes its own upper limit on the vertical sizes of turbulent eddies, so that the $H$-dependence plays no role provided that $H$ is much larger than the stratification limit. ${ }^{1}$ This is precisely the idea justifying the above design. From the physical point of view, it is analogous to the so-called " $z$-less stratification" concept widely used in meteorology, in particular, in the context of the Monin-Obukhov similarity theory.

The latter employs, instead of $R i$, the dimensionless height $z / L$ based on the Obukhov length scale:

$$
L \equiv \frac{(-\tau)^{3 / 2}}{\beta F}
$$

(where $\tau=\overline{u^{\prime} w^{\prime}}$ and $F=\overline{\rho^{\prime} w^{\prime}}$ are the vertical turbulent fluxes of momentum and density), and states that dimensionless characteristics of the surface-layer turbulence are universal functions of $z / L$ (see Monin and Yaglom 1971). Then the static stability is characterised in equal rights by either $R i$ or $z / L$, with the single-valued correspondence between these parameters, whereas $R i$ monotonically increases with increasing $z / L$.

For example, the eddy viscosity, $K_{M} \equiv-\tau / S$, in the surface layer is expressed as $K_{M}=k(-\tau)^{1 / 2} z / \Phi_{M}(z / L)$ where $k$ is the von Karman constant and $\Phi_{M}(z / L)$ is a universal function representing the stability dependence of the ratio of $K_{M}$ to its neutral stability value $K_{M \text { (neutral) }}=k(-\tau)^{1 / 2} z$, which is why $\Phi_{M}(0)=1$ by definition. In these terms, the $z$-less stratification concept postulates that turbulence in very stable stratification (at $z / L \gg 1$ ) no longer depends on $z$ just because the stable stratification imposes a limit on the vertical sizes of turbulent eddies. In the above example it is required that $K_{M}=k(-\tau)^{1 / 2} z / \Phi_{M}(z / L)$ should become independent on $z$ at $z / L \gg 1$, which in turn requires that asymptotically $\Phi_{M}$ should be a linear function: $\Phi_{M} \rightarrow C_{u} z / L$ (where $C_{u}$ is an empirical dimensionless constant). The range of applicability of the $z$-less stratification concept is then determined as $z \gg L$.

In the standard design for the direct numerical simulation (DNS) of turbulence in homogeneous stably-stratified flows, the depth of the computational domain, $H$, plays the same role (of an eddy-size limit) as the distance above the surface, $z$, in surface-layer turbulence. This means that DNS, as well as large-eddy simulation (LES), in domain of depth $H$ is capable of realistically reproducing the homogeneous stably-stratified turbulence only at $L / H \ll 1$. Otherwise the simulated turbulence with the prescribed $R i$ is factually controlled by $H$ and therefore reproduces an almost neutrally-stratified flow in a limited domain. It is conceivable that a series of runs for larger and larger $H$ would reveal essential $H$-dependence of the simulated turbulence until $H$ considerably exceeds $L$. Admittedly, it is too computationally expensive to perform DNS for very large $H$. LES of weakly stable turbulence in very deep domains is also expensive but quite feasible (we are planning such work in the future).

\section{Energy-Budget Arguments}

It seems that the above restrictions are to some extent overlooked by the modern DNS community. In particular, it usually goes without saying that DNS data on the $R i$-depen-

\footnotetext{
1 The simulated turbulence depends also on the horizontal sizes of the domain, which should be taken essentially larger than $H$ to guarantee that the side walls do not impose additional restrictions on the horizontal extension of turbulent eddies.
} 
dence of the turbulent Prandtl number, $P r_{T} \equiv K_{M} / K_{\rho}$ (where $K_{\rho} \equiv-\beta F / N^{2}$ is the eddy diffusivity), are equally grounded for any stratification including very small $R i$. Recently a highly professional and skillful analysis by Venayagamoorthy and Stretch (2006, 2010), focused on the $R i$-dependence of $\operatorname{Pr}_{T}$ at small $R i$, serves as a dramatic example of such common practice.

Its weakness (namely, neglecting the $H$-dependence inevitably essential at small $R i$ ) is demonstrated below employing the turbulent kinetic energy (TKE) budget equation for the stationary homogeneous stably-stratified turbulence-precisely the regime assumed in the above DNS design:

$$
-\tau S+\beta F-\varepsilon_{K}=0,
$$

where $-\tau S>0$ is the TKE generation by the velocity shear, $-\beta F<0$ is the rate of its consumption by work against the stable stratification, and $\varepsilon_{K}>0$ is the rate of TKE viscous dissipation. It follows from Eq. 3 that $\beta F$ cannot exceed $-\tau S$. The ratio of these terms, called the "flux Richardson number", is

$$
R i_{f} \equiv \frac{-\beta F}{-\tau S}
$$

and increases with increasing stability but cannot exceed unity. Hence it has a finite limit:

$$
R i_{f} \rightarrow R_{\infty}=\text { constant }<1 .
$$

This yields the following strong-static-stability asymptotes for the turbulent Prandtl number:

$$
\operatorname{Pr}_{T}=\frac{R i}{R i_{f}} \rightarrow \frac{R i}{R_{\infty}}
$$

and the velocity shear:

$$
S \rightarrow \frac{-\beta F}{-R_{\infty} \tau}=\frac{(-\tau)^{1 / 2}}{R_{\infty} L}
$$

Interpolating between Eq. 7 and the neutral-stability wall law, $S=(-\tau)^{1 / 2} / k z$, yields:

$$
S=\frac{(-\tau)^{1 / 2}}{k z}\left(1+\frac{k}{R_{\infty}} \frac{z}{L}\right)
$$

where $k \approx 0.4-0.5$ and $R_{\infty} \approx 0.2-0.25$, so that $k / R_{\infty} \approx 2$. Recall that the same relation, namely $S=\left[(-\tau)^{1 / 2} / k z\right]\left(1+C_{u} z / L\right)$, was long ago derived from the concept of $z$-less stratification and confirmed empirically with $C_{u} \approx 2$ (e.g., Zilitinkevich and Esau 2007). The above new derivation clarifies its physical meaning and expresses $C_{u}$ through the fundamental constants $k$ and $R_{\infty}$.

Substituting Eq. 8 for $S$ into the definition of the flux Richardson number, Eq. 4, specifies $R i_{f}$ as a monotonically increasing function of $z / L$ :

$$
R i_{f}=\frac{k z / L}{1+k R_{\infty}^{-1} z / L} .
$$

Then, as follows from the first relation in Eq. 6: $P r_{T}=R i_{f}^{-1} R i$, the proportionality factor $\left(R i_{f}^{-1}\right)$ between $\operatorname{Pr}_{T}$ and $R i$

- is infinite in the neutral stability limit (at $z / L=0$ or $R i=0$ ),

- monotonically decreases with increasing $z / L$ or $R i$, 
- $\quad$ and tends to its minimal value, $R_{\infty}^{-1}$, in the very strong stability limit.

Qualitatively, results from DNS of weakly stratified turbulence, in particular the $R i$-dependence of $\mathrm{Pr}_{T}$ shown in Fig. 4 of Venayagamoorthy and Stretch (2010), are consistent with these conditions. This is not surprising: any monotonically increasing function linking the neutral stability limit: $\left.P r_{T}\right|_{R i=0}=P r_{0} \approx 0.7-0.8$ with the large $R i$ limit: $P r_{T}=R_{\infty}^{-1} R i$ (e.g., the simple interpolation: $P r_{T}=P r_{0}+R_{\infty}^{-1} R i$ ) would also look reasonable. However, as follows from the above analysis, direct numerical simulations in question inevitably underestimate the effect of stratification at small $R i$, which is why the interval $0<R i<0.25$ of the curve $\operatorname{Pr}_{T}$ versus $R i$ in the above figure can hardly be considered as grounded. To cover this interval, data on stably-stratified boundary-layer turbulence (although heterogeneous but basically satisfying local energy balances) would be much more suitable.

\section{Concluding Remarks}

The widely used design of direct numerical simulation of stationary homogeneous stablystratified turbulence for given height-constant values of the gradient Richardson number, $R i$, Eq. 1, is physically consistent for the ultimate condition that the height of the computational domain, $H$, is at least an order of magnitude larger than the stratification limit quantified by the Obukhov scale $L$, Eq. 2. Using Eq. 9, this condition becomes

$$
\frac{H}{L}>\frac{10 R_{\infty}}{k} \approx 5 \text {. }
$$

Relying upon the empirical relationship between $R i$ and $z / L$ presented in Fig. 3 of Zilitinkevich and Esau (2007), the above condition corresponds to $R i>0.25$. In other words, the design in question in its present form is justified only for $R i>0.25$.

One more, almost trivial, condition deserves mentioning. With increasing stability the ratio of the horizontal to the vertical sizes of turbulent eddies increases, which is why numerical experiments for large $R i$ should be performed in domains with widths exceeding $10 H$.

Acknowledgements This work has been supported by the EC FP7 projects ERC PBL-PMES (No. 227915) and MEGAPOLI (No. 212520). The author thanks Antti Hellsten and Igor Esau for discussions.

Open Access This article is distributed under the terms of the Creative Commons Attribution Noncommercial License which permits any noncommercial use, distribution, and reproduction in any medium, provided the original author(s) and source are credited.

\section{References}

Gerz T, Schumann U, Elghobashi SE (1989) Direct numerical simulation of stratified homogeneous turbulent shear flows. J Fluid Mech 200:563-594

Monin AS, Yaglom AM (1971) Statistical fluid mechanics, vol 1. MIT Press, Cambridge, 769 pp

Schumann U, Gerz T (1995) Turbulent mixing in stably stratified shear flows. J Appl Meteorol 34:33-48

Venayagamoorthy SK, Stretch DD (2006) Lagrangian mixing in decaying stably stratified turbulence. J Fluid Mech 564:197-226

Venayagamoorthy SK, Stretch DD (2010) On the turbulent Prandtl number in homogeneous stably stratified turbulence. J Fluid Mech 644:359-369

Zilitinkevich S, Esau I (2007) Similarity theory and calculation of turbulent fluxes at the surface for the stably stratified atmospheric boundary layers. Boundary-Layer Meteorol 125:193-296 